\title{
Menetrier's disease associated with anaemia and venous thromboembolism in a 53 year old male
}

\begin{abstract}
Ménétrier's disease (MD) is a rare hyperproliferative protein-losing, pre-malignant gastropathy of the gastric foveolar epithelium. We present an unusual case of $H$. Pyloriinduced MD complicated by vascular thrombosis. A 53 year old Caucasian male with no significant past medical history presented with a 2 week history of shortness of breath on exertion. Routine bloods revealed a Hemoglobin of $6 \mathrm{~g} / \mathrm{dL}(13-17 \mathrm{~g} / \mathrm{dL})$ with an MCV of 64 fL (80-100fL). Iron studies confirmed an iron deficiency anemia. Other notable lab values included serum albumin $1.9 \mathrm{~g} / \mathrm{dL}(3.5-5.0 \mathrm{~g} / \mathrm{dL})$ with no proteinuria
\end{abstract}

Gastroscopy showed grossly abnormal, thickened gastric folds in the body and multiple polypoid lesions in the antrum. CT Imaging of the thorax, abdomen and pelvis revealed a grossly dilated stomach, with diffuse circumferential wall thickening, measuring up to $3 \mathrm{~cm}$ in maximum diameter. It also revealed extensive bilateral $2^{\text {nd }}$ order pulmonary emboli and a large IVC clot extending from the level of the renal veins to the common iliac veins. Antral biopsies revealed a moderate, chronic gastritis positive for $H$. Pylori. There was no evidence of malignancy but foveolar hyperplasia and cystically dilated foveolar glands consistent with Menetrier's Disease.

The patient was transfused, given therapeutic anti-coagulation and triple therapy for $H$. Pylori. At 12 months follow-up, the patient remains asymptomatic with $\mathrm{Hb} 14 \mathrm{~g} / \mathrm{dl}$ and serum albumin now $3.2 \mathrm{~g} / \mathrm{dL}$.

Keywords: Menetrier's disease, gastric foveolar hyperplasia, H. Pylori, hypertrophic gastritis
Volume 10 Issue 2 - 2019

\section{Barra Neary, Carthage Moran, Glen Doherty}

St Vincent's University Hospital, Ireland

Correspondence: Dr Barra Neary, St Vincent's University

Hospital, Dublin, Ireland, Tel +353879566 I2I,

Email barraneary@svhg.ie

Received: February II, 2018 | Published: March 15, 2019

\section{Introduction}

Ménétrier Disease (MD) is a rare hyperproliferative proteinlosing, pre-malignant gastropathy of the gastric foveolar epithelium. First described by Pierre Menetrier in $1888,{ }^{1}$ the exact annual incidence is uncertain due to some confusion in the literature. It appears to be an acquired disorder of uncertain aetiology that typically involves the fundus and body of the stomach but spares the antrum. It is characterized by expansion of epithelial cells that constitute the surface mucous cell compartment. It can be complicated by anaemia, hypochlorhydria, oedema and occasionally venous thromboembolism.

\section{Case presentation}

In this instance, a 53 year old Caucasian male with no significant past medical history presented to his primary care provider with a 2 week history of shortness of breath on exertion. Physical exam was unremarkable. Routine bloods revealed a Hemoglobin of $6 \mathrm{~g} / \mathrm{dL}$ (13$17 \mathrm{~g} / \mathrm{dL})$ with an MCV of $64 \mathrm{fL}(80-100 \mathrm{fL})$. Iron studies confirmed an iron deficiency anaemia. Other notable lab values included serum albumin $1.9 \mathrm{~g} / \mathrm{dL}(3.5-5.0 \mathrm{~g} / \mathrm{dL})$ with no proteinuria. CT Imaging of the thorax, abdomen and pelvis revealed a grossly dilated stomach, with diffuse circumferential wall thickening, measuring up to $3 \mathrm{~cm}$ in maximum diameter (Figure 1). It also revealed extensive bilateral $2^{\text {nd }}$ order pulmonary emboli and a large IVC clot extending from the level of the renal veins to the common iliac veins (Figure 2).

With an iron deficiency anaemia and a concern for an occult GI bleed, endoscopy was performed that showed grossly abnormal, thickened gastric folds in the body and multiple polypoid lesions in the antrum with scattered blood throughout (Figures 3) (Figure 4).

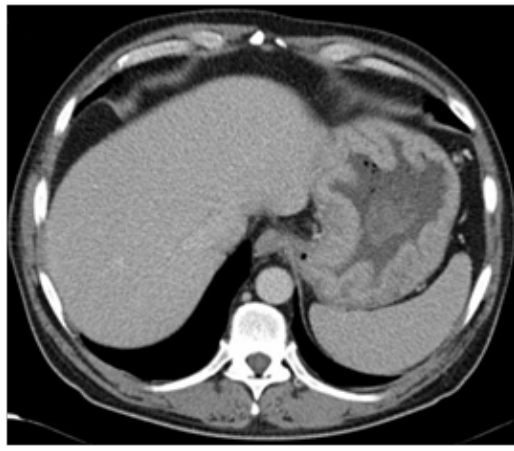

Figure I CT image of grossly dilated stomach, with diffuse circumferentia wall thickening.

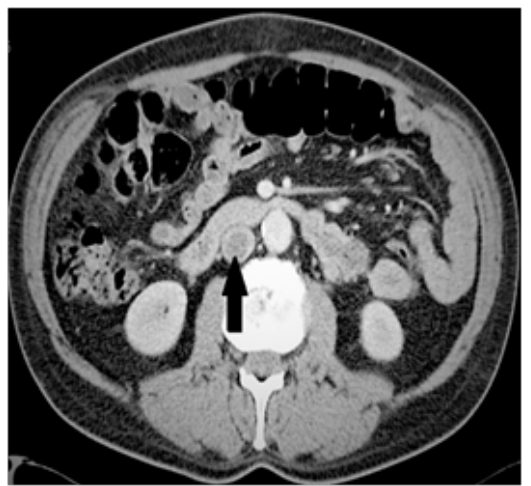

Figure 2 CT image with IVC thrombus. 


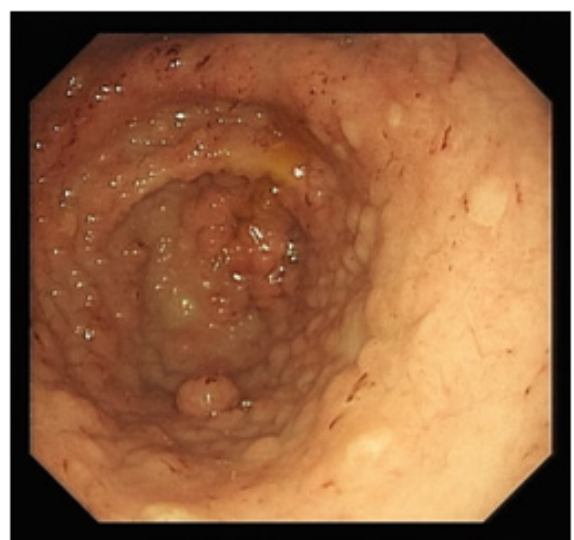

Figure 3 Polypoid lesions in the antrum.

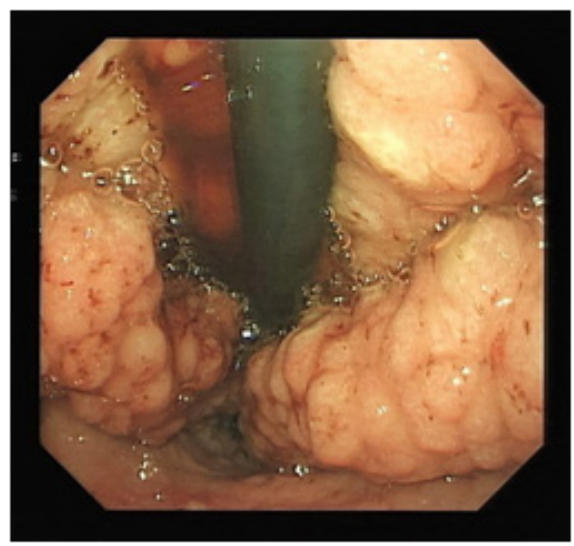

Figure 4 Polypoid lesions in fundus.

Colonoscopy was essentially normal with one $3 \mathrm{~cm}$ polyp at the hepatic flexure. With concern for a diffuse neoplastic process, endoscopic ultrasound was performed which showed diffuse nodular thickened gastric fold pattern with hypertrophied gastric mucosal layers with preservation of gastric wall layers indicating a benign disorder, no cystic appearance.

Antral biopsies revealed a moderate, chronic gastritis positive for H. Pylori (Figure 5). There was no evidence of malignancy but foveolar hyperplasia and cystically dilated foveolar glands consistent with Menetrier's Disease (Figure 6).

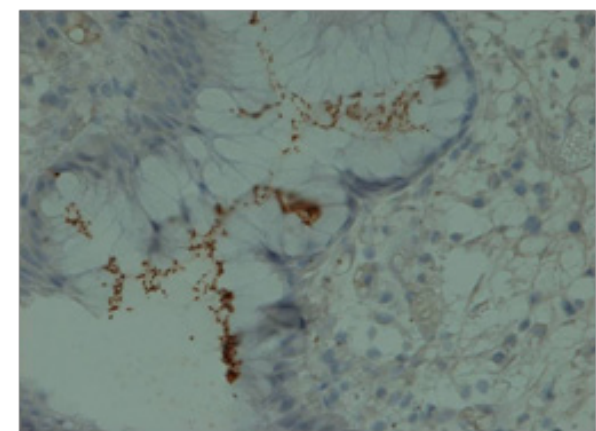

Figure 5 Antral biopsies staining positive for H. Pylori.

The patient was transfused, given therapeutic anti-coagulation and triple therapy for H. Pylori. At 12 months follow-up, the patient remains asymptomatic with $\mathrm{Hb} 14 \mathrm{~g} / \mathrm{dl}$ and serum albumin now $3.2 \mathrm{~g} /$ dL

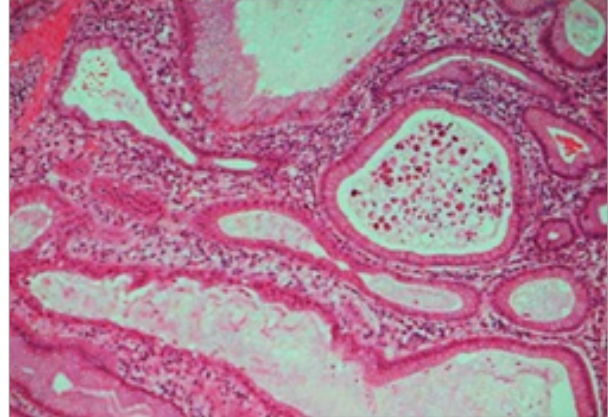

Figure 6 Antral biopsies showing cystically dilated foveolar glands.

\section{Discussion}

In $\mathrm{MD}$, the average age at diagnosis in adults is 55 , and the disease tends to be more common in men than in women. The disease most commonly presents with epigastric pain (65\%), fullness and vomiting $(38 \%)$ and generalized peripheral oedema with hypoalbuminemia $(80 \%) .^{2}$ Diagnosis of MD requires clinical and morphological correlation with the endoscopic findings. MD can be difficult to establish with routine endoscopic biopsies as hyperplastic gastric polyps can have identical histological features in small biopsy samples; deeper "snare," or full-thickness, biopsies of the gastric mucosa are recommended. Giant rugal oedematous folds are seen on gastroscopy often with antral sparing. Radiologically, the wall of the gastric body and fundus is diffusely thickened. Histology of biopsy material shows diffuse foveolar hyperplasia with cystic dilatation of the glandular portion of the gastric mucosa. There is often a certain degree of inflammation in the lamina propria, though the enlarged folds can develop superficial ulcers with an influx of neutrophils into the lamina propria. There can also be an accumulation of eosinophils in the lamina propria and they are occasionally the predominant inflammatory cell in the lamina propria. ${ }^{3}$ Previously thought to be of uncertain aetiology, MD is now thought to involve increased signalling of the epidermal growth factor receptor (EGFR), which results in local overproduction of TGF-a and proliferation of epithelial cells of the mucous cell compartment. Animal models have shown that increased exposure to TGF-a leads to mucosal cell proliferation and overexpression in the stomach specifically has led to an MDphenotype in mice. ${ }^{4,5}$ Patients with MD have also exhibited enhanced immunoreactivity for TGF- $\alpha$ in their expanded surface mucous cell compartment. ${ }^{6}$ However, the precise underlying molecular defect that results in upregulation of TGF- $\alpha$ in Ménétrier disease is not known. It is probable that there is host susceptibility to developing Ménétrier disease, with a genetic predisposition toward developing this disorder. Previous studies show the coexistence of MD with infections, such as $\mathrm{CMV}^{7} \mathrm{HSV}^{8}$ and H. Pylori. ${ }^{9}$ There is little in the literature of hypercoagulability in the setting of MD with no underlying malignancy or other risk factors, ${ }^{10}$ there is an increased incidence of thrombotic events likely due to decreased intravascular volume or loss of anti-thrombotic proteins as in nephrotic syndrome. As MD is considered a pre-malignant condition with risk of gastric carcinoma of 6-10\%, recognizing it and monitoring progression is of paramount importance, with some authors recommending endoscopic surveillance every one to two years for early detection of dysplasia or carcinoma. ${ }^{11}$ Treatment is based on eradication of the potential underlying aetiologies with other options available including BD PPI therapy, cetuximab (a monoclonal antibody that blocks ligand binding to the EGFR) with gastrectomy as the optimum therapeutic procedure in rare instances of refractory disease. 


\section{Acknowledgments}

None.

\section{Conflicts of interest}

There is no conflict of interest to disclose.

\section{References}

1. Ménétrier P. Gastric polyadenomas and their relationship to stomach cancer. Arch Physiol Norm Pathol. 1888;1:32-55;236-262

2. Meuwissen SG, Ridwan BU, Hasper HJ, et al. Hypertrophic proteinlosing gastropathy. A retrospective analysis of 40 cases in The Netherlands. The Dutch Menetrier Study Group. Scand J Gastroenterol Suppl. 1992;194:1-7.

3. Wolfsen HC, Carpenter HA, Talley NJ. Ménétrier's disease: a form of hypertrophic gastropathy or gastritis? Gastroenterology. 1993;104(5):1310-1319.

4. Rutten MJ, Dempsey PJ, Solomon TE, et al. TGF-alpha is a potent mitogen for primary cultures of guinea pig gastric mucous epithelial cells. Am J Physiol. 1993;265(2 pt 1):G361-G369.
5. Sharp R, Babyatsky MW, Takagi H, et al. Transforming growth factor alpha disrupts the normal program of cellular differentiation in the gastric mucosa of transgenic mice. Development. 1995;121(1):149-161.

6. Bluth RF, Carpenter HA, Pittelkow MR, et al. Immunolocalization of transforming growth factor-alpha in normal and diseased human gastric mucosa. Hum. Pathol. 1995;26(12):1333-1340.

7. Occena RO, Taylor SF, Robinson CC, et al. Association of cytomegalovirus with Ménétrier's disease in childhood: report of two new cases with a review of literature. J Pediatr Gastroenterol Nutr. 1993;17(2):217-224.

8. Jun DW, Kim DH, Kim SH, et al. Ménétrier's disease associated with herpes infection: response to treatment with acyclovir. Gastrointestinal Endosc. 2007;65(7):1092-1095.

9. Ishikawa T, Ando T, Obayashi H, et al. Helicobacter pylori isolated from a patient with Ménétrier's disease increases hepatocyte growth factor mRNA expression in gastric fibroblasts: comparison with Helicobacter pylori isolated from other gastric diseases. Dig Dis Sci. 2008;53(7):1785.

10. McDermott VG, Connolly CE, Finnegan P, et al. Menetrier's disease presenting with deep venous thrombosis, iron deficiency anaemia and early evolution to atrophic gastritis. Ir J Med Sci. 1986;155(2):53-54.

11. Nalle SC, Turner JR. Menetrier's disease therapy: rebooting mucosal signaling. Sci Transl Med. 2009;1(8):8ps10. 